\title{
THERMOMECHANICAL STRESSES IN A SOLID SPHERE IN CONDITIONS OF PULSED HEATING OVER THE SURFACE.
}

\author{
T. Kartvelishvili", M. Yumashev ${ }^{* *}$
}

\begin{abstract}
The object of investigation of this work is brittle materials under conditions of rapid local heating, which leads to large temperature gradients in the sample and in turn to large mechanical stresses that can cause cracking of the sample and a significant deterioration in its strength and wear resistance. In this problem, an approximate method for calculating unsteady temperature fields is considered, when there is a rapid and intensive heating of a solid sphere by the action of a heat flux, described by its density, which is constant in time. The existence of the fusion front is not taking into account. The method of approximate solution of the linear heat equation is based on the idea of a thermal front. Analytic formulas are obtained that determine the temperature dependence on the coordinate and on time. Then the comparison of the approximate and numerical solution of the heat conductivity equation is made. Investigation of the stress field is carried out to determine the most probable areas of the occurrence of cracks. Analytic expressions are obtained in the case of an elastic material.
\end{abstract}

Keywords: thermal front, heat flux, brittle materials, laser beam, stress distribution.

\section{Introduction}

For bodies it is necessary to consider temperature and stress distributions in order to explain the various processes that arise in them under thermal action. We use approximate solutions for the temperature distribution, introducing the concept of the thermal front, additional boundary conditions on it and integral methods of heat balance, which are used in the works (Yumashev, 2010 and Kuudinov, 2011). The practicality of approximate solutions and the possibility of their refinement can be advantageous over exact solutions, since these solutions are difficult to obtain and they appear in the form of infinite series. In this paper, the temperature distribution is given by a polynomial of the second degree. Moreover, this method makes it much faster and easier to obtain solution in comparison with the numerical method.

\section{Methods}

Over the surface of the sphere a uniformly distributed constant heat flux begins to flow at the initial moment of time.

\subsection{Mathematical statement of the problem}

We have the heat equation in a spherical coordinate system, where everything depends only on the radial coordinate:

$$
\frac{\partial T(\mathrm{r}, \mathrm{t})}{\partial t}=a^{2} \frac{1}{r^{2}} \frac{\partial}{\partial r}\left(\frac{\partial T(\mathrm{r}, \mathrm{t})}{\partial r}\right),
$$

Initial and boundary conditions of the problem are:

\footnotetext{
Graduade Student Tatiana Kartvelishvili: Lomonosov Moscow State University, Leninskie Gory 1; 119234, Moscow; Russia, tania.urban@yandex.ru

** $\quad$ Assistant Prof. Mikhail Yumashev, Ph.D.: Lomonosov Moscow State University, Leninskie Gory 1; 119234, Moscow; Russia, yumashevmikhail@gmail.com
} 


$$
\left\{\begin{array}{l}
t=0: \mathrm{T}(\mathrm{r}, 0)=\mathrm{T}, 0 \leq \mathrm{r} \leq \mathrm{R} \\
r=0: \frac{\partial T(0, t)}{\partial r}=0, t>0 \\
r=R: \lambda \frac{\partial T(R, t)}{\partial r}=q_{0}, t>0
\end{array},\right.
$$

where $R$ is a radius of the sphere, $q_{0}$ is a heat flux density.

\subsection{Temperature distribution}

Let us find an approximate solution based on the concept of the thermal front. Thermal front is a certain mathematical surface, which is the boundary between heated and cold parts of the body. It can be interpreted as a temperature line, value of which is practically indistinguishable from the initial temperature. Such a boundary exists, because any measuring device has limited accuracy of measurements. We need to solve:

$$
L(T)=\frac{\partial T(\mathrm{r}, \mathrm{t})}{\partial t}-a^{2} \frac{1}{r^{2}} \frac{\partial}{\partial r}\left(\frac{\partial T(\mathrm{r}, \mathrm{t})}{\partial r}\right)=0
$$

The first boundary condition shifts from the center of the sphere to the thermal front and the initial condition is modified:

$$
\left\{\begin{array}{l}
r=l(t): \mathrm{T}(1, \mathrm{t})=\mathrm{T}_{0}, \mathrm{t}>0 \\
r=l(t): \frac{\partial T(1, t)}{\partial r}=0, t>0 \\
r=R: \lambda \frac{\partial T(R, t)}{\partial r}=q_{0}, t>0 \\
t=0: l(0)=R
\end{array}\right.
$$

Let us obtain the solution in the form of a polynomial of the second degree:

$$
T(r, t)=A_{0}(t)+A_{1}(t) \mathrm{r}+A_{2}(t) r^{2},
$$

by solving simultaneous equations:

$$
T(r, t)=\left\{\begin{array}{l}
T_{0}+\frac{q_{0}}{\lambda} \frac{(l-R)^{2}}{2(R-l)}, l(t) \leq r \leq R \\
T_{0}, 0 \leq r \leq R
\end{array}\right.
$$

It is necessary to determine the temperature front as a function of time. We use the integral heat balance method described in the paper (Yumashev, 2010). We require the following integral expression to be satisfied:

$$
\int_{V} L(T) d V=0 \Leftrightarrow \int_{l(t)}^{R} r^{2}\left[\frac{\partial T(\mathrm{r}, \mathrm{t})}{\partial t}-a^{2} \frac{1}{r^{2}} \frac{\partial}{\partial r}\left(r^{2} \frac{\partial T(r, \mathrm{t})}{\partial r}\right)\right] d r=0
$$

Using $l(0)=R$, we get a front-time dependence:

$$
l^{4}+R l^{3}+R^{2} l^{2}-9 R^{3} l+6 R^{4}=60 R^{2} a^{2} t
$$

In this approximation the thermal front does not depend on the density of the heat flux, but we can get the time at which the thermal front reaches the center of the sphere. Substituting $l\left(t_{0}\right)=0$, we obtain:

$$
t_{0}=\frac{1}{10} \frac{R^{2}}{a^{2}}=\frac{1}{10} \frac{R^{2} c \rho}{\lambda}
$$

In order to find the temperature distribution after the indicated moment of time, we again approximate the exact solution of the heat equation by a square polynomial. Unknown coefficients can be found from the system: 


$$
\left\{\begin{array}{l}
r=0: \mathrm{T}(0, \mathrm{t})=\mathrm{T}_{c}(\mathrm{t}), \mathrm{t}>t_{0} \\
r=0: \frac{\partial T(0, t)}{\partial r}=0, t>t_{0} \\
r=R: \lambda \frac{\partial T(R, t)}{\partial r}=q_{0}, t>t_{0} \\
T_{c}\left(t_{0}\right)=T_{0}
\end{array}\right.
$$

Solving it and using again the integral heat balance method:

$$
T=a^{2} \frac{q_{0}}{\lambda} \frac{3}{R}\left(t-t_{0}\right)+T_{0}+\frac{q_{0}}{\lambda} \frac{1}{2 R} r^{2}
$$

Let us compare the approximate and numerical solutions of the heat conduction equations for the case of a sphere of steel. To obtain a numerical solution of the heat equation, we use the finite difference method and apply an implicit four-point scheme. The program is written in $\mathrm{C}++$. Input parameters are: $R=0.01$ $\mathrm{m}, \lambda=46 \mathrm{~W} /\left(\mathrm{m}^{*} \mathrm{C}\right), \rho=7800 \mathrm{~kg} / \mathrm{m}^{3}, c=460 \mathrm{~J} /\left(\mathrm{kg}^{*} \mathrm{C}\right), T_{0}=20^{\circ} \mathrm{C}, q_{0}=10^{7} \mathrm{~W} /\left(\mathrm{m}^{2}\right)$. We consider our temperature approximations only until the temperature on the outer surface of the sphere reaches the melting temperature, so for steel $T_{m}=1450-1520^{\circ} \mathrm{C}$.

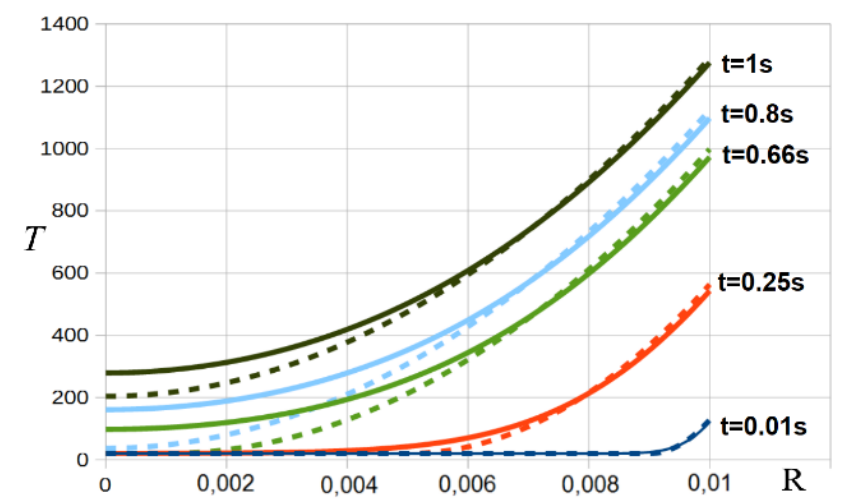

Fig. 1: Temperature distribution (solid curve is a numerical solution, dotted is an approximate). The initial stage corresponds to the right-hand end of the figure.

We see that at such a high flux density, which is realized when metal is processed by a laser, for example, the maximum difference in the exact and approximate temperature is $100{ }^{\circ} \mathrm{C}$. The minimal difference is manifested in the initial stages of the action.

\subsection{Stress distribution}

We consider the problem of determining the stress-strain state of an elastic sphere in the presence of a temperature field and a free external boundary. The problem is considered in a quasi-static formulation. The equilibrium equations for a sphere in a spherical coordinate system in the absence of inertial forces and body forces are transformed into a single equilibrium equation:

$$
\frac{d \sigma_{r}}{d r}+\frac{2}{r}\left(\sigma_{r}-\sigma_{t}\right)=0
$$

Knowing the generalized Hooke law at a variable temperature (the Duhamel-Neumann ratio), we write the relationship between stresses and strains. Then, solving the equilibrium equation, we obtain:

$$
u=\alpha \frac{(1+v)}{(1-v)} \frac{1}{r^{2}} \int_{0}^{r} T(r) r^{2} d r+C_{1} r+\frac{C_{2}}{r_{2}}
$$

Constants are found from the boundary conditions: in the center of the sphere there are no displacements ( $u=0, r=0)$, the outer surface of the sphere is free of effort $\left(\sigma_{r}=0, r=R\right.$ ).

Final expressions for stresses are: 


$$
\begin{gathered}
\sigma_{r}=\left\{\begin{array}{l}
\left\{\begin{array}{l}
\frac{\alpha E}{(1-v)} \frac{q_{0}}{\lambda} \frac{1}{(\mathrm{R}-1)}\left(\frac{l^{2}}{3}-\frac{l R}{2}+\frac{R^{2}}{5}-\frac{l^{5}}{30 R^{3}}\right), 0 \leq \mathrm{r}<1 \\
\frac{\alpha E}{(1-v)} \frac{q_{0}}{\lambda} \frac{1}{(\mathrm{R}-1)}\left(\left(\frac{l^{2}}{3}-\frac{l R}{2}+\frac{R^{2}}{5}-\frac{l^{5}}{30 R^{3}}\right)-\left(\frac{l^{2}}{3}-\frac{l r}{2}+\frac{r^{2}}{5}-\frac{l^{5}}{30 r^{3}}\right)\right), 1 \leq \mathrm{r} \leq \mathrm{R}
\end{array}, t \leq t_{0}=\frac{1}{10} \frac{R^{2}}{a^{2}}\right. \\
\frac{2 \alpha E}{(1-v)} \frac{q_{0}}{\lambda} \frac{1}{2 R} \frac{1}{5}\left(\mathrm{R}^{2}-\mathrm{r}^{2}\right), t>t_{0}=\frac{1}{10} \frac{R^{2}}{a^{2}}
\end{array}\right. \\
\sigma_{t}=\left\{\begin{array}{l}
\left\{\begin{array}{l}
\frac{\alpha E}{(1-v)} \frac{q_{0}}{\lambda} \frac{1}{(\mathrm{R}-1)}\left(\frac{l^{2}}{3}-\frac{l R}{2}+\frac{R^{2}}{5}-\frac{l^{5}}{30 R^{3}}\right), 0 \leq \mathrm{r}<1 \\
\frac{\alpha E}{(1-v)} \frac{q_{0}}{\lambda} \frac{1}{(\mathrm{R}-1)}\left(2\left(\frac{l^{2}}{3}-\frac{l R}{2}+\frac{R^{2}}{5}-\frac{l^{5}}{30 R^{3}}\right)+\left(\frac{l^{2}}{3}-\frac{l r}{2}+\frac{r^{2}}{5}-\frac{l^{5}}{30 r^{3}}\right)-(1-\mathrm{r})^{2}\right), 1 \leq \mathrm{r} \leq \mathrm{R} \\
\frac{2 \alpha E}{(1-v)} \frac{q_{0}}{\lambda} \frac{1}{2 R} \frac{1}{5}\left(\mathrm{R}^{2}-2 \mathrm{r}^{2}\right), t>t_{0}=\frac{1}{10} \frac{R^{2}}{a^{2}}
\end{array}, t \leq t_{0}=\frac{1}{10} \frac{R^{2}}{a^{2}}\right.
\end{array}\right.
\end{gathered}
$$
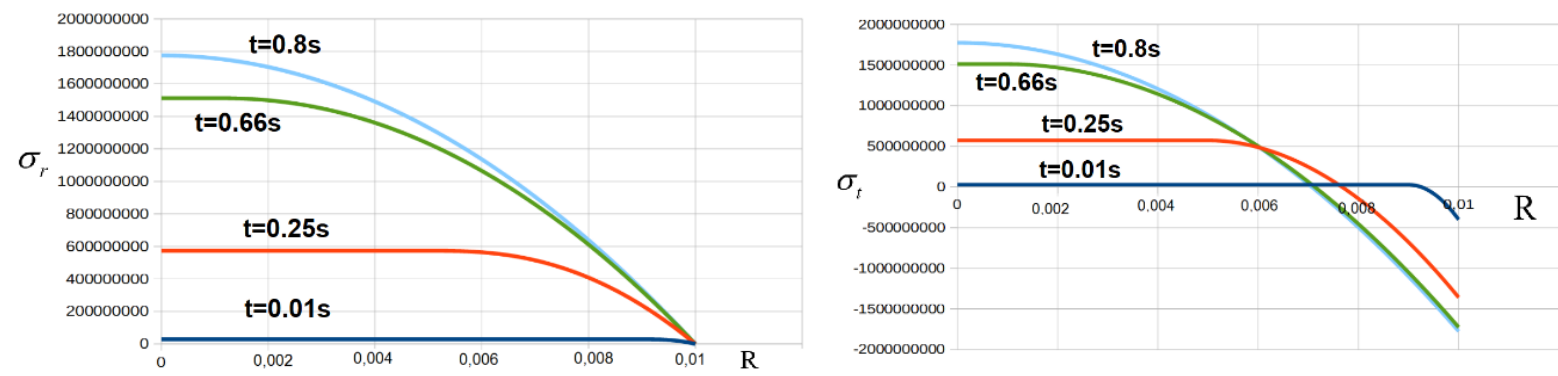

Fig. 2: Radial (on the left) and circumferential (on the right) stress distributions.

In Fig. 2 we find that the maximum tensile stresses appear at the center of the sphere at the moment when $t_{0}=0.7$ seconds. The maximum compressive stresses also appear at the time $t_{0}=0.7 \mathrm{~s}$, but on the outer surface of the sphere and they are equal $1.775 \cdot 10^{9} \mathrm{~Pa}$. It is known that for most materials the greatest danger is represented by shear stresses (Andryushchenko, 2012). An estimate of the maximum shift gives $\tau_{\max }=\sigma_{r}-\sigma_{t}$. Then we obtain that the maximum shear stresses appear on the outer surface of the sphere when $t_{0}=0.7 \mathrm{~s}$.

\section{Conclusions}

Numerical-analytical solutions are obtained for temperature and stress distributions in a quasistatic formulation for problems that do not take into account melting. The analysis of the maximum shear stresses, which are the greatest danger for most materials, is performed - the maximum occurs on the outer surface of the sphere.

\section{References}

Andryushchenko, V. and Goloveshkin, V. (2012) Estimation of temperature stresses arising in a meteoroid during hypersonic motion in the earth's atmosphere. Problems of Computational and Applied Mechanics, MAX Press, pp. 80-85, Moscow (in Russia).

Kuudinov, I. (2011) Mathematical modeling of heat conduction and hydrodynamics processes numerically by analytical methods on the basis of using additional boundary conditions. Samara (in Russian).

Yumashev, M. and Yumasheva, M. (2010) Modeling of the process of heating the body with intense thermal action on the surface. Vestnik Moskovskogo Universiteta, Moscow (in Russia). 